This is the post print version of the article, which has been published in Logopedics Phoniatrics Vocology. 2015, 40(1), 14-23. https://doi.org/10.3109/14015439.2013.775333

This document has been downloaded from TamPubutafi The Insitutional Repository of University of Tampere

\title{
Human vocal tract resonances and the corresponding mode shapes investigated by three-dimensional finite-element modelling based on CT measurement
}

Tomáš Vampola ${ }^{a}$, Jaromír Horáček ${ }^{b 1}$, Anne-Maria Laukkanen ${ }^{c}$, Jan G. Švec ${ }^{d}$

${ }^{a}$ Department of Mechanics, Biomechanics and Mechatronics, Faculty of Mechanical Engineering, Czech Technical University in Prague, Technická 4, 16607 Prague 6, Czech Republic, e-mail: tomas.vampola@fs.cvut.cz

${ }^{b}$ Institute of Thermomechanics, Academy of Sciences of the Czech Republic, Dolejškova 5, 18200 Praha 8, Czech Republic, e-mail: jaromirh@it.cas.cz

' Speech and Voice Research Laboratory, School of Education, University of Tampere, FIN 33014 Tampere, Finland, e-mail: Anne-Maria.Laukkanen@uta.fi

${ }^{d}$ Department of Biophysics, Faculty of Sciences, Palacký University Olomouc, 17. listopadu 12,77146 Olomouc, e-mail: SvecJanG@gmail.com

running headline:

\section{Human vocal tract resonances and 3D mode shapes}




\begin{abstract}
Resonance frequencies of the vocal tract have traditionally been modelled using onedimensional models. These cannot accurately represent the events in the frequency region of the formant cluster around 2.5-4.5 kHz, however. Here, the vocal tract resonance frequencies and their mode shapes are studied using a three-dimensional finite element model obtained from computed tomography measurements of a subject phonating on vowel [a:]. Instead of traditional five, up to eight resonance frequencies of the vocal tract were found below the prominent antiresonance around $4.7 \mathrm{kHz}$. The three extra resonances were found to correspond to modes which were axially asymmetric and involved piriform sinuses, valleculae and transverse vibrations in the oral cavity. The results therefore suggest that the phenomenon of speaker's and singer's formant clustering may be more complex than originally thought.
\end{abstract}

Keywords: biomechanics of human voice, voice production modelling, acoustic mode shapes of vibration, speaker's and singer's formant 


\section{Introduction:}

Cavities of the vocal tract play an important role for the timbre of human voice. It is known that the two lowest resonance frequencies of the vocal tract are responsible for creating the formants $F_{1}$ and $F_{2}$, which mainly determine vowels, see e.g. (1-2). The role of the third formant F3, in distinguishing vowels and vowel categories, varies between languages; see e.g. (3-4). In general, the higher vocal tract resonances, forming the formants $F_{3}$ and higher, contribute to the individual voice quality. While the study of vowels has received a lot of attention in the past, the research on voice timbre, related to the upper formants, has been more limited. It has focused on voice quality in classical or other types of singing (5-6) and in speakers (7-9). Recently, the role of upper formants has been studied in relation to the effects of vocal exercising and voice therapy applying phonation into a tube or a straw. In our former study, we have shown that the shape of the vocal tract does change during and after performing vocal exercises by phonating into the so called resonance tube (10).

This study builds upon the former study and addresses the following questions: "Which vocal tract resonances and how many of them play a role in the resonant voice quality after vocal exercising?" and "Which mode shapes are responsible for these resonances?” We can note that a formant can be understood as an excited acoustic resonance.

Traditionally, five formants have been described in the frequency range up to $5 \mathrm{kHz}$. These five formants have traditionally been studied using the simple one dimensional (1D) models of the vocal tract created by concatenated cylindrical or conical tubes. Such models are very useful in analyzing the acoustic resonances of the vocal tract when the longitudinal plane wave propagation is the dominant mechanism for transferring the source signal from the vocal folds to the lips, see e.g. $(1,2,11)$. However, at frequencies over $3 \mathrm{kHz}$ the simple $1 \mathrm{D}$ models of the vocal tract cannot fully capture the acoustic phenomena: the acoustic mode shapes of vibrations may become axially asymmetric and transverse modes can arise, which may influence the individual timbre of human voice (12-14). The formants around $3 \mathrm{kHz}$ are important for individual characteristics of professional voices, i.e. especially for speakers and singers. A cluster of resonances around 2-3 kHz, i.e., the so-called "singer's formant cluster", has been found in classically trained (male) singing voice $(5,7,15)$, and a cluster between 3 and $4 \mathrm{kHz}$ has been described to build up the so called “actor's or speaker's formant cluster" in a good speaking voice $(7,8,16)$.

New methods of mathematical modeling enable to model the voice production in the higher frequency range using more realistic three-dimensional (3D) models. Following the pioneering studies of, e.g. Lu et al. (17), and Motoki (13), such models were recently 
developed, e.g. by Vampola et al. $(10,14)$ using the finite element (FE) method, or by Takemoto et al. (18) using the finite difference modeling approach. Experimentally the higher frequency range up to $10 \mathrm{kHz}$ was studied on physical models of the vocal tract for Japanese vowels by Honda et al. (19) and Kitamura et al. (20). These authors were mainly engaged in description of dips in the resonant characteristics of the vocal tract due to the existence of the piriform sinuses and valleculae and transversal modes in the mouth cavity.

Here, we present FE modeling of the acoustic mode shapes of vibration and the radiated pressure in front of the mouth focusing on the effects of nonsymmetrical 3D acoustic modes of vibration in the frequency range up to $8 \mathrm{kHz}$. The FE models of the vocal tract were created from the Computerized Tomography (CT) measurements.

\section{Material and Method:}

\section{CT and acoustic measurements:}

The CT data were obtained using the Light Speed VCT GE - 64 (General Electric) device. A trained female speaker volunteered as subject of the study. She signed a consent form allowing the CT examination to be performed. In order to obtain the best representative samples of the speaker's most resonant speaking voice, the CT scanning was carried out after the subject had been phonating for some minutes into a resonance tube (made of glass, length $28 \mathrm{~cm}$, inner diameter $7 \mathrm{~mm}$ ), which aimed at reaching the best-possible speaking voice quality. When performing the examination, the subject was given a sign through the CT intercom system to start phonating. The subject performed the phonation into the tube first and then immediately continued by phonating on the vowel [a:] for ca. $4 \mathrm{~s}$ without the tube. The CT scanning occurred immediately after the subject started the second phonation. The midsagittal and corresponding coronal and transversal slices obtained from the CT measurements are shown in figure 1.

Insert Figure 1 somewhere here

The sound was captured by a miniature microphone (DPA Alleroed, Denmark, type 4066-F, omnidirectional) fixed to the subject's nose and recorded using a digital audio recorder (MAudio, Microtrack II). The acoustic analysis was done using the software Multi-Speech by Kay Pentax (Model 3700, v. 3.2.0.). The signal was low-pass filtered with the cut-off frequency of $6000 \mathrm{~Hz}$ and downsampled to the sampling rate of 12000 samples per second. Then, LPC formant analysis was performed tracking the formants during the course of the two repeated sustained vowels (frame length $25 \mathrm{~ms}$, frame advance $10 \mathrm{~ms}$, autocorrelation 
analysis method using a $16^{\text {th }}$ filter order, Blackman window and pre-emphasis of 0.9 ). The LPC numerical data were exported as text files. Since the ambient noise in the CT room (signal to noise ratio $9.8 \mathrm{~dB}$ ) caused random disturbances in tracking the LPC formants, the approximate average frequency of each of the formants was evaluated by the maxima of histograms with $10 \mathrm{~Hz}$ bins created and plotted using the software Sigma Plot 2002 for Windows (v. 8.02) (see figure 8 later). More details on the CT and acoustic measurements can be found in (10), where the same subject was studied before, during and after the vocal exercising.

\section{Construction of the volume and finite element models:}

The recorded CT images were first segmented and processed into 3D volume models of the vocal tract. The developed volume model is shown in figure 2, where the epilaryngeal airways, the piriform sinuses and the valleculae are clearly visible. Nasal cavities were not included in the model since the velar port was found to be closed in this subject after the resonant exercises, thus preventing the sound entering the nasal tract $(10,21)$.

\section{Insert Figure 2 somewhere here}

After meshing the volume models, the 3D FE model of the vocal tract was constructed within the framework of the program ANSYS. The model consisted of about 182000 tetrahedral acoustic finite elements and is shown in figure 3a. The speed of sound $350 \mathrm{~m} / \mathrm{s}$, the air density $1.2 \mathrm{~kg} / \mathrm{m}^{3}$ and hard walls inside the vocal tract were considered for simplicity. In order to simulate the radiation of the sound out of the mouth, the human head was modelled approximately as a sphere with the radius of ca. $10 \mathrm{~cm}$, and the surrounding air was modelled as a set of finite elements filling in the space between the head and an outer sphere with the radius of ca. $20 \mathrm{~cm}$ (figure $3 \mathrm{~b}$ ). The outer sphere was modelled by so-called infinite, nonreflecting elements FLUID130. The acoustic space between the spheres was modelled by about 630000 finite elements with an average element size of ca. $5 \mathrm{~mm}$.

Figure 3c shows a simplified fully symmetric FE model of the vocal tract created from the volume model using the cross-sectional areas measured from the vocal folds to the lips. This model simulates frequently used 1D models of the vocal tract, see e.g. $(2,22,23)$.

Insert Figure 3 somewhere here 
Transient analysis, acoustic modal analysis and harmonic analysis of the FE model:

In order to determine the transfer function of the vocal tract, the supraglottal spaces of the FE model were excited at the level of the vocal folds by a very short impulse of acoustic airflow volume velocity covering a broad band frequency range from zero up to about $10 \mathrm{kHz}$ with the input decrease of ca. $0.5 \mathrm{~dB}$ (see figure 4). The transient analysis was performed with the time step $\Delta t=1 \times 10^{-5} \mathrm{~s}$ and the total simulated time was $T=0.4 \mathrm{~s}$.

Insert Figure 4 somewhere here

In order to find how the resonance peaks and the minima of the transfer function are related to the mode shapes of the acoustic vibrations in the vocal tract, an acoustic modal analysis was performed on the FE model. Since the modal analysis does not easily allow including the spaces outside the vocal tract, zero acoustic pressure at the lips was assumed for simplicity in this case. The vocal tract was considered to be closed at the level of the vocal folds and therefore no influence of the trachea was modelled.

Finally, the harmonic analysis of the full FE model, including the radiating space outside the vocal tract, was used for a detailed study of the mode shapes of vibrations inside the vocal tract. These were excited at the resonant and selected antiresonant frequencies at the vocal folds position by the harmonic signal corresponding to the airflow velocity with unit amplitude $1 \mathrm{~m}^{3} / \mathrm{s}$.

\section{Results:}

Vocal tract resonances - the transfer function:

The acoustic pressure and the spectrogram of the response computed at the distance of $10 \mathrm{~cm}$ in front of the mouth using the full 3D FE model of the vocal tract (shown in figure 3b) are presented in time domain in figure 5. The spectrogram of the response shows decreasing amplitudes of higher harmonics in the signal with increasing time, which is due to the higher radiation losses for the higher frequencies. The computed power spectral density (PSD) is shown in figure $6(\mathrm{PSD}=\mathrm{FFT}(\mathrm{P}) * \mathrm{FFT}(\mathrm{P}) / \mathrm{N}$, where FFT(P) is the Fourier transform of the pressure $\mathrm{P}(t), \mathrm{N}$ is the number of samples and * denotes the complex conjugated value. The analysis was performed for $\mathrm{N}=40000$ and the sampling frequency $100 \mathrm{kHz}$ resulting in the frequency resolution of $\Delta f=2.5 \mathrm{kHz}$ ). The formation of a cluster of resonances $\mathrm{R}_{4}-\mathrm{R}_{8}$ in the region of the so called "speaker's formant" $(7,8))$ is clearly visible around $4 \mathrm{kHz}$ followed by a deep anti-resonance at about $4740 \mathrm{~Hz}$.

Insert Figure 5 somewhere here 
Insert Figure 6 somewhere here

For a comparison, figure 7 shows the spectrogram and the power spectral density of the acoustic response computed at the lips using the simplified symmetric "1D" model of the vocal tract (shown in figure 3c). In this case, no formant cluster is created, only five resonances exist up to $5 \mathrm{kHz}$ with mode shapes that correspond to the classical $1 \mathrm{D}$ longitudinal acoustic waves, and no transversal modes are excited here due to the full symmetry of the model.

\section{Insert Figure 7 somewhere here}

In order to compare the resonance frequencies of the vocal tract model with the real formant frequencies, an LPC analysis of the vowel produced during the CT evaluation was performed. The histogram maxima corresponding to the most frequently occurring LPC formant frequencies were taken as the representative formant frequencies for the analyzed vowel (figure 8). The results of the acoustic LPC analysis for the fundamental $\left(\mathrm{F}_{0}\right)$ and formant $\left(\mathrm{F}_{1}\right.$ $\mathrm{F}_{4}$ ) frequencies are summarized in Table I and compared with the computed values. The difference between the calculated resonance frequencies and the measured formant frequencies is less than $8 \%$ which is acceptable, considering the noisy acoustic sample. This comparison provides the evidence that the FEM computations are realistic. No attempt was made here to compare the LPC and FEM results for the higher formants, since the LPC method was not prepared for calculating the possible acoustic effects of the side branches of the vocal tract that appear to play an increasingly important role for the higher formants in the FEM results (see further). Figures $5 \mathrm{~b}$ and 6 show that the FEM-computed resonance frequencies $R_{1}-R_{12}$ are within the frequency range from zero up to $6 \mathrm{kHz}$ and in total 18 resonance frequencies can be found in the frequency range up to $8 \mathrm{kHz}$.

Insert Figure 8 somewhere here

Insert Table I somewhere here

Vocal tract modes - acoustic eigenmodes and resonance modes responsible for producing the resonance peaks:

The computed acoustic mode shapes corresponding to the resonance frequencies $R_{1}-R_{12}$ are shown in figure 9. The first three eigenmodes for the resonance frequencies $R_{1}-R_{3}$ are the classical fundamental longitudinal modes with one, two and three nodes (recognizable as zero amplitudes in the figure) along the vocal tract length, see e.g. (2). These are similar to the modes occurring in a simple tube when the propagation of the longitudinal waves in the vocal 
tract is dominant and when the acoustics characteristics of the vocal tract can be described by a simple 1D acoustic theory, see e.g. (14). The higher eigenmodes for the resonance frequencies $\mathrm{R}_{4}-\mathrm{R}_{12}$ are, however, already three-dimensional, asymmetric or transversal and are not associated with the sound waves propagation of a symmetrical plane wave in a cylindrical duct. The resonance $\mathrm{R}_{4}$ still reminds the classic longitudinal mode with four nodes along the vocal tract length but important vibration amplitudes are seen here in the valleculae cavities, where the vibrations are in antiphase with the vibrations in the laryngeal cavity. The resonance $\mathrm{R}_{8}$ then reminds of the classic longitudinal mode with five nodes along the vocal tract length but here we see, in addition, large vibration amplitudes in both the piriform sinuses and valleculae with some left-right asymmetry. The most important transversal vibrations in the oral cavity are seen for the resonances $R_{5}, R_{11}$ and $R_{12}$. The remaining four resonances $R_{6}, R_{7}, R_{9}$ and $R_{10}$ have the dominant antinodes of the pressure situated in the piriform sinuses, valeculae and laryngeal ventricles, where these parts of the vocal tract are vibrating with maximal amplitudes in phase or antiphase and with relatively low pressure amplitudes in the oral cavity. The higher eigenmodes belonging to the resonances $R_{13}-R_{18}$ in the frequency region above $6 \mathrm{kHz}$ are not shown in the figure anymore - they were found to be very complicated in 3D space, because both the longitudinal and transversal modes in the oral cavity and in the lower pharynx were combined here.

\section{Insert Figure 9 somewhere here}

Figure 10 compares the harmonically excited acoustic mode shapes of vibration at the resonant frequencies for the resonances $R_{8}$ and $R_{9}$ and at the main antiresonance frequency ( $f_{\mathrm{A}}=4743 \mathrm{~Hz}$ ) situated between them (see figure 6). On the left it shows the absolute values of the pressure inside the vocal tract and on the right there are the corresponding phase shifts between the excitation signal and the response. While for the resonance $\mathrm{R}_{8}$ a relatively high level of the acoustic pressure can be seen near the lips (567 Pa), for the resonance $\mathrm{R}_{9}$ and for the antiresonance the pressure level in the oral cavity is near zero. In all three cases, the highest values of the pressure are in the laryngeal cavity, in the piriform sinuses and in the valleculae. The high drop in the maximal values of the pressure inside the vocal tract (from $5061 \mathrm{~Pa}$ for the resonance $\mathrm{R}_{8}$ down to $358 \mathrm{~Pa}$ for the resonance $\mathrm{R}_{9}$, see figure 10) corresponds to the substantial decrease of the pressure level in front of the mouth (see also figure 6). The driven maximal pressure amplitudes inside the vocal tract for the resonance $\mathrm{R}_{9}$ (358 $\mathrm{Pa}$ ) and for the antiresonance (566 Pa) are comparable.

\section{Insert Figure 10 somewhere here}




\section{Discussion:}

Instead of the traditional five resonance peaks, we have found eight resonance peaks in the transfer function of the 3D model of the vocal tract in the frequency region from zero up to $5 \mathrm{kHz}$. Besides of the five resonances $\left(\mathrm{R}_{1}-\mathrm{R}_{4}\right.$ and $\left.\mathrm{R}_{8}\right)$ resembling the well known longitudinal modes, three additional modes were detected. Two of the resonances $\left(R_{6}\right.$ and $\left.R_{7}\right)$ showed large influence of the side branches of the vocal tract, mainly the piriform sinuses, which are normally disregarded in the simple $1 \mathrm{D}$ models. The last additional resonance peak $\left(\mathrm{R}_{5}\right)$ was found to be related to the transverse mode in the oral cavity.

The piriform sinuses have been previously described as being mainly responsible for the vocal tract antiresonance peak which occurs as the spectral minimum around $5 \mathrm{kHz}(18-20)$. The current data show, however, that the piriform sinuses can also produce spectral maxima at frequencies below this antiresonance. These can positively contribute to the build-up of the formant cluster in the frequency range of 3-5 kHz. This agrees with the Sundberg study (5) on generation of the singer's formant peak considering wide piriform sinuses as one of the necessary conditions which must be met. In technical terms, the piriform sinuses act as the "pole-zero" pair. The amplification of the spectral components at the frequencies below the spectral minima could also be detected in the results of other authors but without receiving specific attention (e.g., $(18,19))$.

Our data also show that the left and right piriform sinus are not symmetric in the examined subject and therefore the resonance peaks split in two (see the acoustic mode shapes for the resonances $R_{6}$ and $R_{7}$ in figure 9 ), occurring as two extra formants in the sound spectrum (see figures $5 \mathrm{~b}$ and 6 ). Furthermore, a closer look at the acoustic modes of vibration in figures 9 and 10 reveals that valleculae are involved in the resonances $R_{4}, R_{8}$ and $R_{10}$, making the resulting modes rather complex. The cluster created by the resonances $R_{4}-R_{8}$ is enhanced by the first transversal mode of vibration $\left(\mathrm{R}_{5}\right)$ which has a low damping and its importance is growing in time (see the spectrogram in figure $5 b$ ).

In this pilot study, we have studied only a single vowel [a:] in a single subject. Interindividual differences in the cavity sizes will change the frequencies of the vocal tract resonances and antiresonances. These will be altered also by different vowels. Further studies are therefore needed to justify the results of the case study presented here. Nevertheless our data provide a strong evidence that: 1 ) the 3D complexity of the vocal tract causes additional formants to appear in the frequency range up to $5 \mathrm{kHz}$ which are not observed in simple 1D models, and 2) the side branches of the vocal tract such as the piriform sinuses and vallecullae do not only cause spectral minima but can become also valuable contributors to the formant 
cluster around 3-5 kHz. Since the human hearing threshold is relatively low around 3-5 kHz $(24,25)$, the sound energy concentration around the 3.5-4 kHz $\left(\mathrm{R}_{4}-\mathrm{R}_{8}\right)$ region can be useful to assure maximum audibility of the produced sound and individual voice timbre. This can play an important role in communication both from the point of view of production and perception.

\section{Conclusion:}

Up to eight vocal tract resonances (formants) were found below $5 \mathrm{kHz}$. The lower resonances $\mathrm{R}_{1}-\mathrm{R}_{3}$ (and to some degree also the resonances $\mathrm{R}_{4}$ and $\mathrm{R}_{8}$ correspond to the classical longitudinal vibration modes, which can be obtained also with $1 \mathrm{D}$ vocal tract models. For higher resonances, however, more complicated asymmetric and transversal 3D modes of vibration are prominent, which require a 3D modelling approach. While piriform sinuses and valleculae cause antiresonances appearing as spectral minima at the frequencies above $5 \mathrm{kHz}$, they also resonate and add energy in the region of the speaker's or singer's formant cluster around 3-5 kHz. As such these side cavities of the vocal tract may serve as valuable contributors to the resulting voice quality.

\section{Acknowledgements}

The research was supported by the grant GACR No. P101/12/1306 and by the Academy of Finland No 106139 Biomechanical study on the traumatizing mechanisms in vocal fold vibration and by the COST Action 2103 Advanced Voice Function Assessment. The research of dr. Švec has been supported by the European Social Fund projects no. CZ.1.07/2.4.00/17.0009 and CZ.1.07/2.3.00/20.0057, The authors are also very grateful to Doc. MUDr. Petr Krupa from the Hospital U Svaté Anny in Brno for providing the CT data of a human vocal tract during phonation.

\section{References}

1. Fant G. Acoustic theory of speech production. The Hague, Paris: Mouton, 1970.

2. Titze IR. Principles of voice production. National Centre for Voice and Speech, Iowa City; 2000. ISBN: 0-87414-122-2.

3. Aaltonen O. The effect of relative amplitude levels of F2 and F3 on the categorization of synthetic vowels. Journal of Phonetics 1985; 13(1):1-9.

4.Vaissière J. On the acoustic and perceptual characterization of reference vowels in a cross-language perspective. Online Proceedings of The 17th International Congress of 
Phonetics Sciences (ICPhS XVII), August 17-21, 2011, Hong Kong, China: http://www.icphs2011.hk/ICPHS_CongressProceedings.htm, pp. 52-59.

5. Sundberg J. Articulatory interpretation of the singing formant. J. Acoust. Soc. Am. 1974; 55: 838-844.

6. Zangger Borch D, Sundberg J. Some phonatory and resonatory characteristics of the rock, pop, soul and Swedish dance band styles of singing. Journal of Voice 2011; 25(5):532537.

7. Leino T. Long-term average spectrum study on speaking voice quality in male actors, In: Friberg A, Iwarsson J, Jansson E, Sundberg J, editors. SMAC93, Proceedings of the Stockholm Music Acoustics Conference, July 28-Aug. 1, 1993. Stockholm: The Royal Swedish Academy of Music, 1994; 79: P. 206-210.

8. Nawka T, Anders LC, Cebulla M, Zurakowski D. The speaker's formant in male voices. Journal of Voice 1997; 11(4): 422-428.

9. Bele I. The speaker’s formant. Journal of Voice 2006;20(4):555-578.

10. Vampola T, Laukkanen AM, Horáček J, Švec JG. Vocal tract changes caused by phonation into a tube: A case study using computer tomography and finite element modeling. J. Acoust. Soc. Am, 2011; 129(1): 310-315.

11. Radolf V. Direct and inverse task in acoustics of the human vocal tract. PhD. thesis, Czech Technical University in Prague. 2010; 95 p. (in Czech).

12. El Masri S, Pelorson X, Saguet P, Badin P. Development of the transmission line matrix method in acoustics applications to higher modes in the vocal tract and other complex ducts. Int J Numer Model 1998; 11(3):133-51.

13. Motoki K. Three-dimensional acoustic field in vocal-tract. Acoust Sci Tech 2002;23(4):207-12.

14. Vampola T, Horáček J, Švec JG. FE modeling of human vocal tract acoustic. Part I: Production of Czech vowels, Acta Acust United Acust 2008; 94(3): 433-447.

15. Titze IR, Story BH. Acoustic interactions of the voice source with the lower vocal tract. J. Acoust. Soc. Am. 1997; 101: 2234-2243.

16. Leino T, Laukkanen AM, Radolf V. Formation of the actor's/speaker's formant: A study applying spectrum analysis and computer modeling. Journal of Voice 2011; 25(2): 150158. 
17. Lu Ch, Nakai T, Suzuki H. Finite element simulation of sound transmission in vocal tract. J.Acoust. Soc. Jpn.(E) 1993; 14(2): 63-72.

18. Takemoto H, Mokhtari P, Kitamura T. Acoustic analysis of the vocal tract during vowel production by finite-difference time-domain method. J. Acoust. Soc. Am. 2010; 128(6): 3724-3738.

19. Honda K, Takemoto H, Kitamura T, Fujita S, Takano S. Exploring human speech production mechanisms by MRI. IEICE TRANS. INF. \& SYST. 2004; E87-D: 5: 10501058.

20. Kitamura T, Takemoto H, Adachi S, Honda K. Transfer functions of solid vocal-tract models constructed form ATR MRI database of Japanese vowel production. Acoust. Sci. Tech. 2009; 30(4): 288-296.

21. Laukkanen AM, Horáček J, Krupa P, Švec JG. The effect of phonation into a straw on the vocal tract adjustments and formant frequencies. A preliminary MRI study on a single subject completed with acoustic results. Biomed. Signal Process. Control 2012; 7(1): 5057.

22. Story BH, Laukkanen AM, Titze IR. Acoustic impedance of an artificially lengthened and constricted vocal tract. Journal of Voice 2000; 14: 455-469.

23. Story BH, Titze IR, Hoffman EA. The relationship of vocal tract shape to three voice qualities, J. Acoust. Soc. Am. 2001; 109: 1651-1667.

24. ANSI S3.4-2007 American National Standard Procedure for the Computation of Loudness of Steady Sounds. Melville, NY: Acoustical Society of America; 2007.

25. Glasberg BR, Moore BCJ. Prediction of absolute thresholds and equal-loudness contours using a modified loudness model. J Acoust Soc Am 2006; 120(2):585-8. 


\section{Figures}
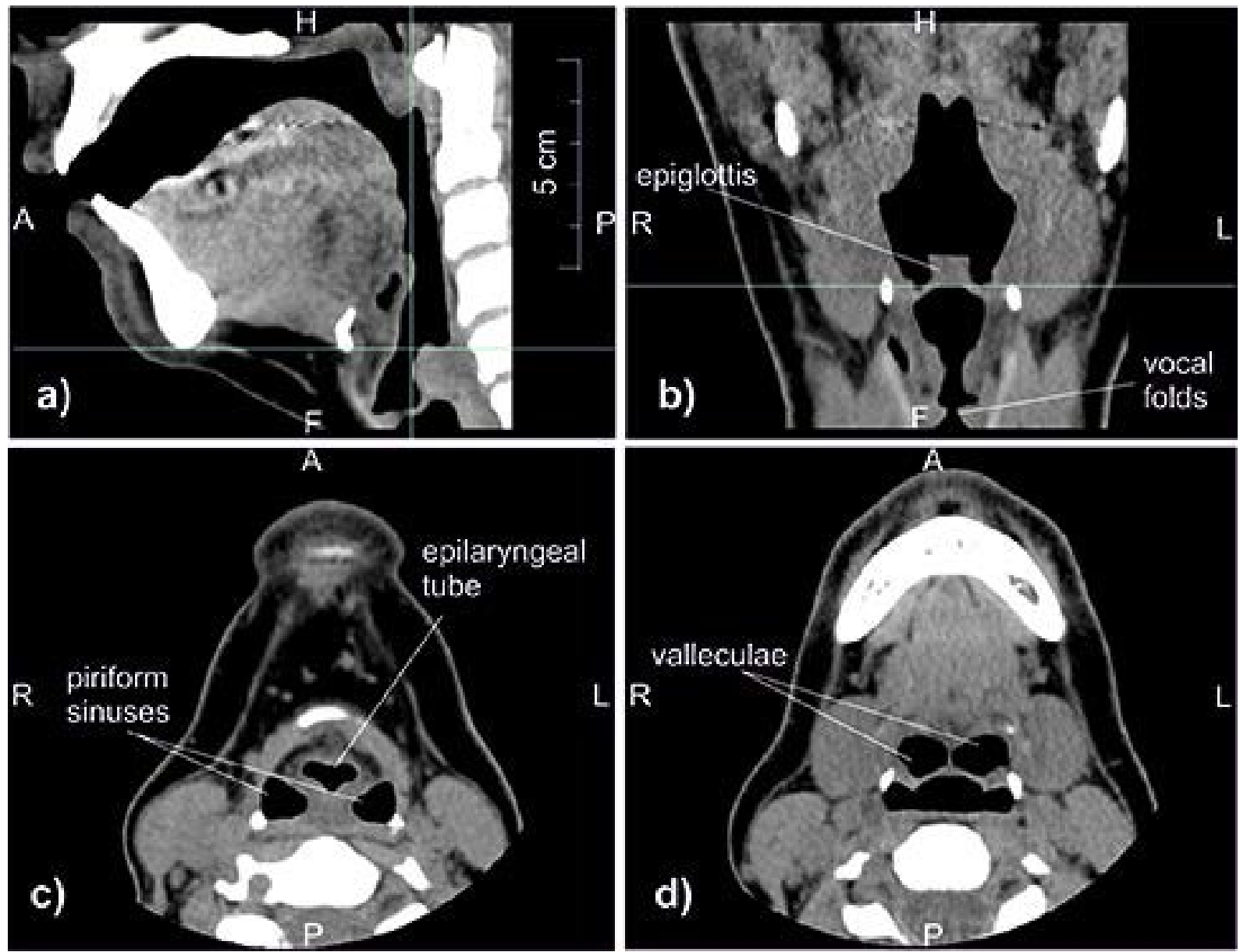

Figure 1. Midsagittal (a), coronal (b) and transversal (c,d) images of the vocal tract for the vowel [a:] obtained from the CT scanning. The horizontal lines in a) and b) show the position for the images in c) and d), respectively. 


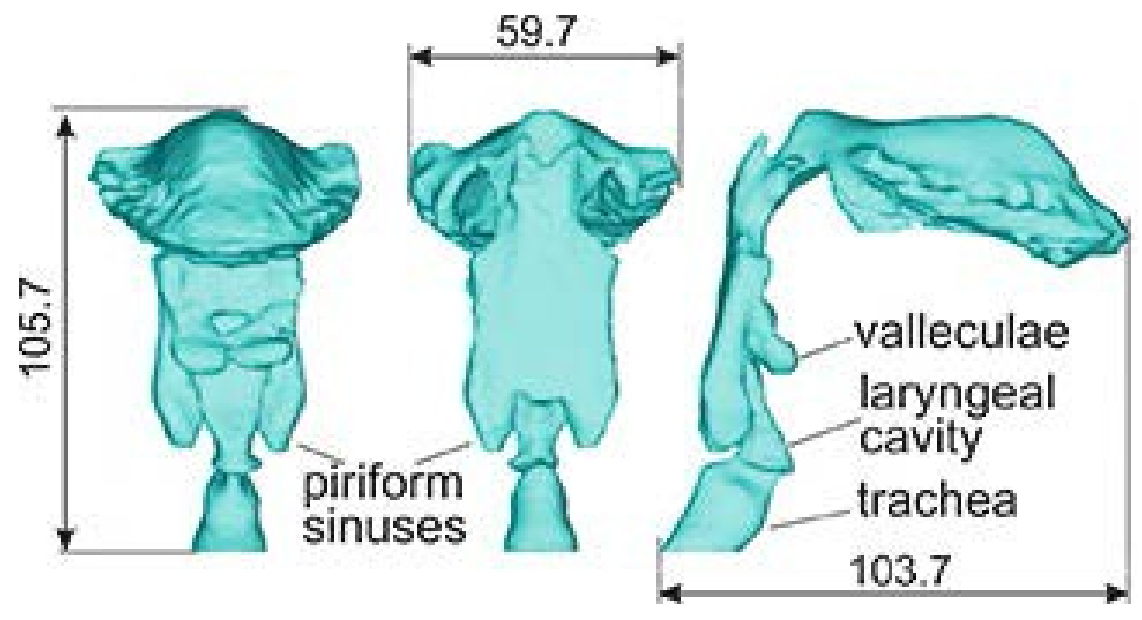

Figure 2. Volume model of the vocal tract for phonation on [a:]. Distances are in mm. 


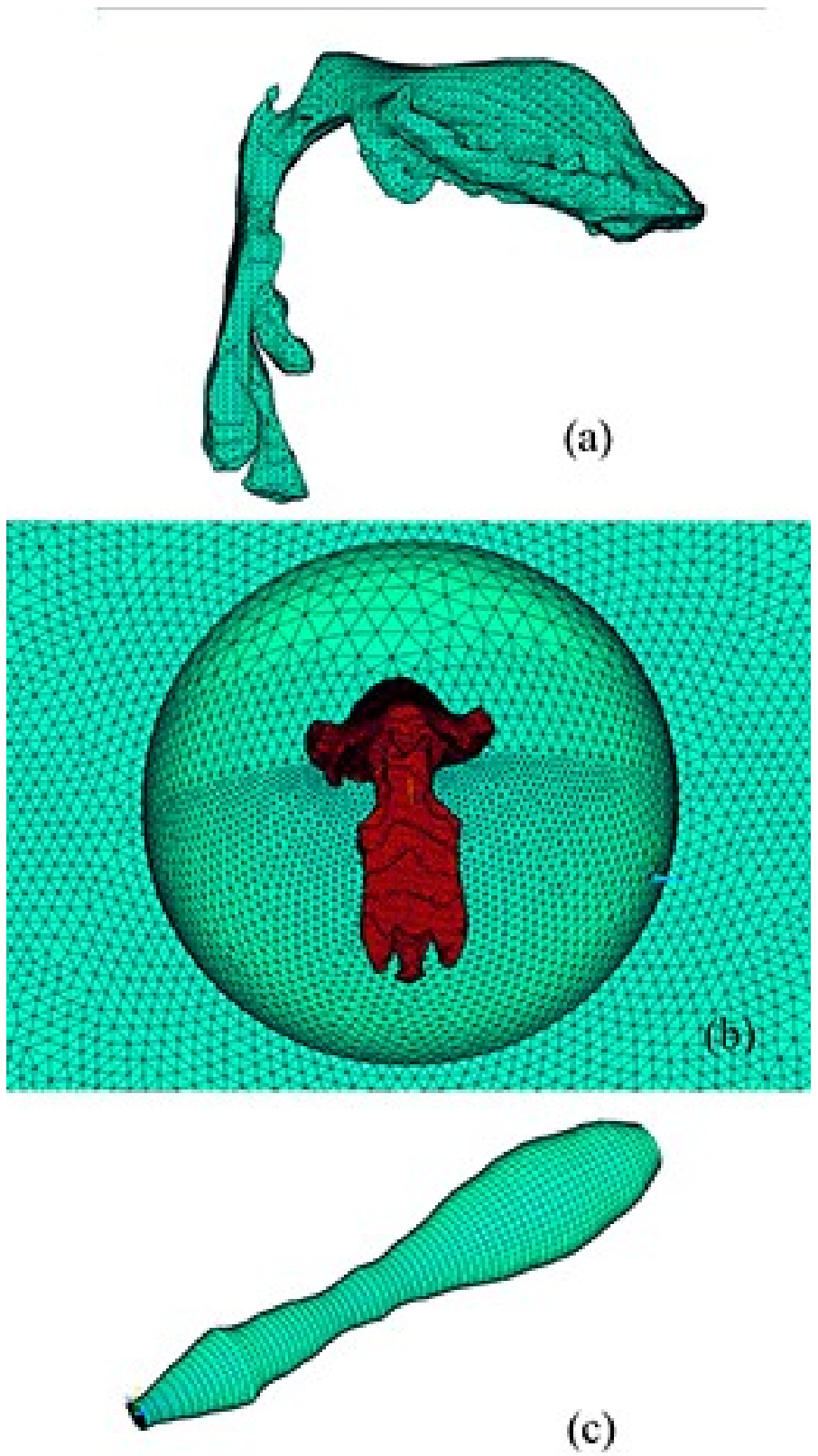

Figure 3. FE models of the vocal tract for the vowel [a:]: a) 3D model constructed from the CT images, b) full 3D model including the surrounding infinite acoustic space used for simulation of sound propagation from the mouth, c) simplified fully symmetric model constructed from the volume model using the cross-sectional areas of measured along the vocal tract from the vocal folds to the lips. 

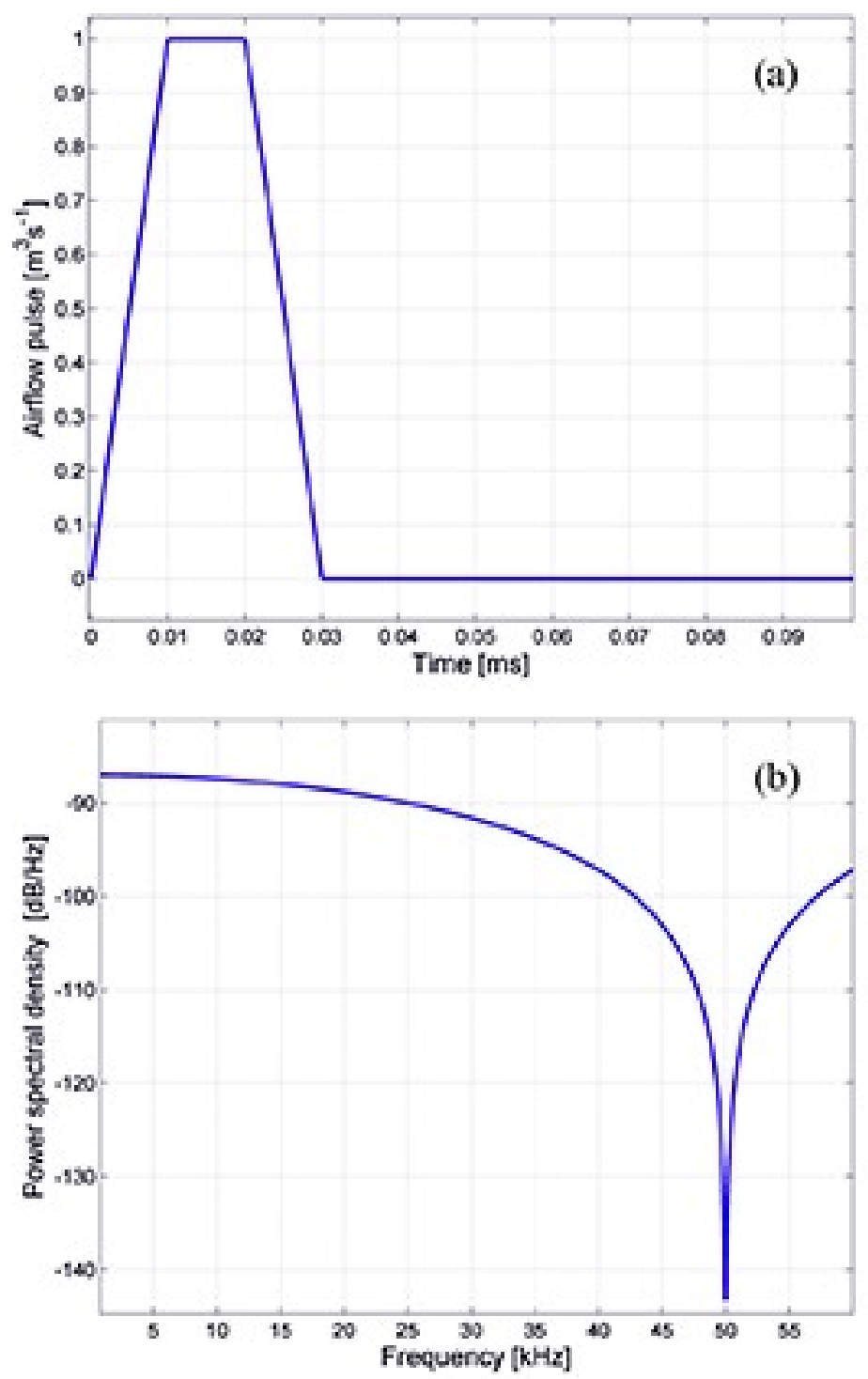

Figure 4. Airflow velocity pulse used for the excitation of the FE model: a) in the time domain, b) in the frequency domain. 

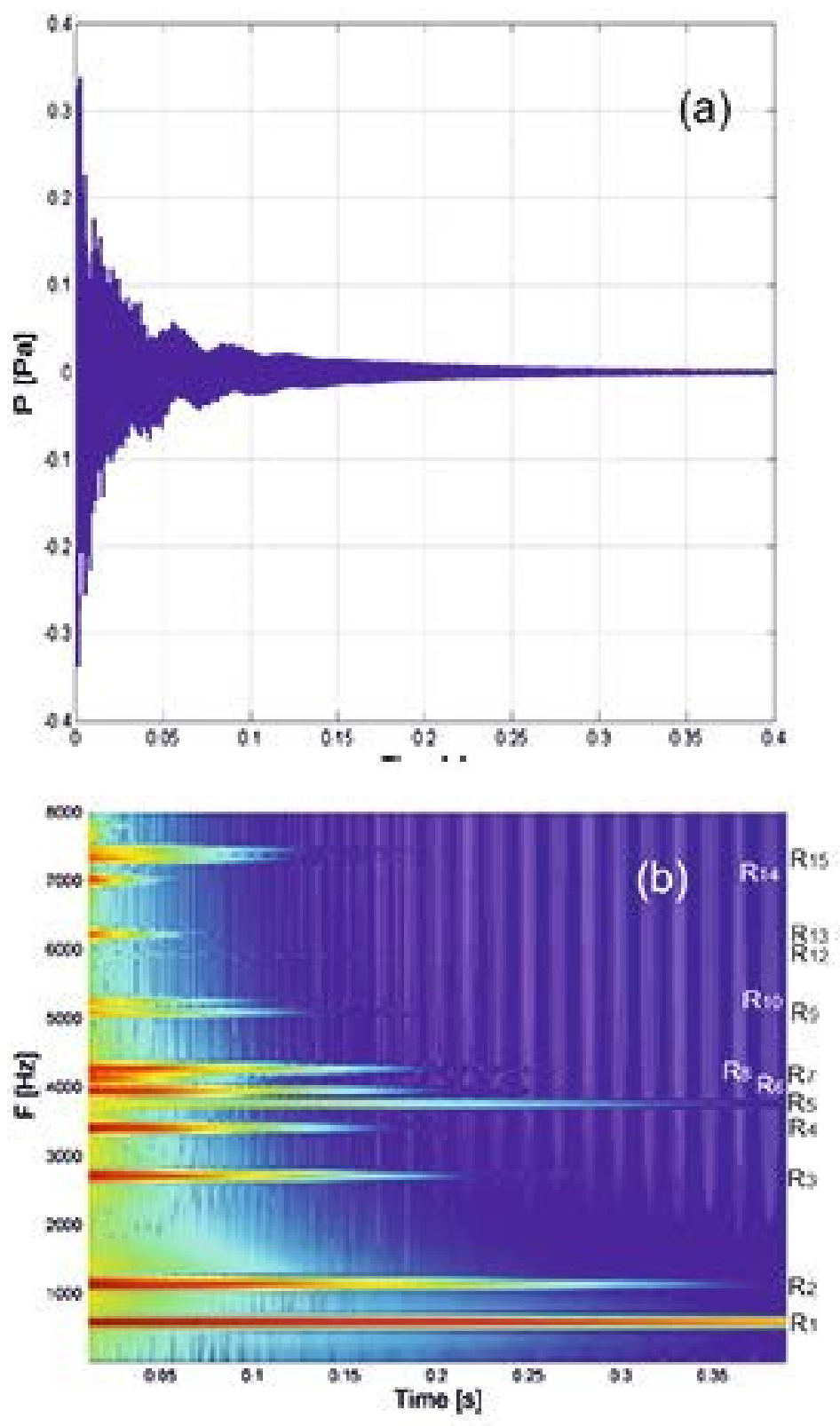

Figure 5. Transient response in time domain of the acoustic pressure computed at the distance of $10 \mathrm{~cm}$ in front of the mouth excited by a short airflow velocity pulse at the vocal folds (upper panel) and numerically simulated spectrogram of the sound signal (lower panel) using the full 3D model of the vocal tract shown in figure 3b. 


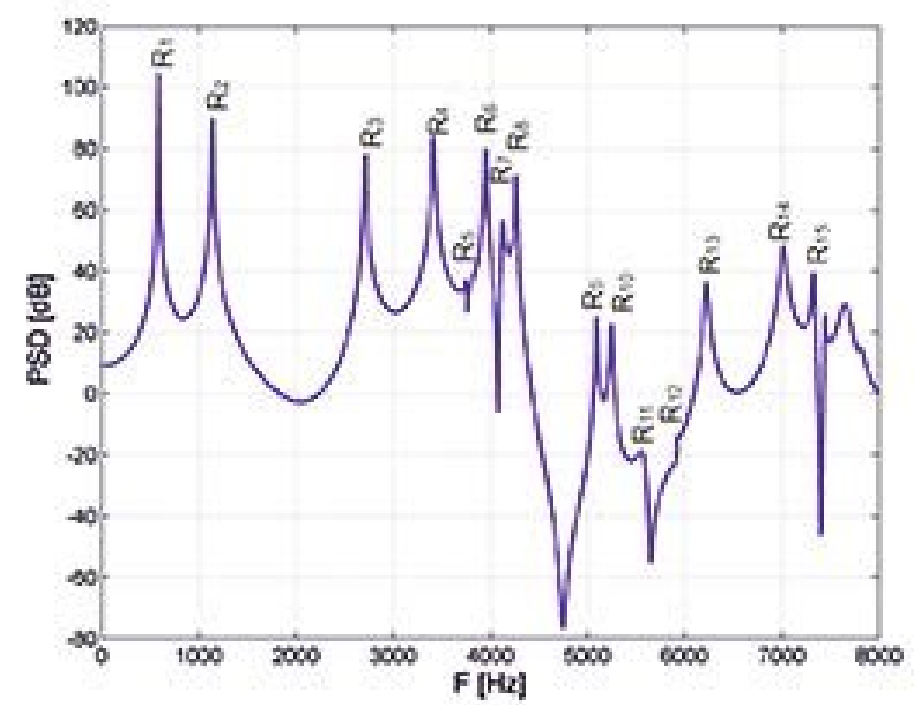

Figure 6. Power spectral density of the acoustic pressure computed at the distance of $10 \mathrm{~cm}$ in front of the mouth excited by a short airflow velocity pulse at the vocal folds using the full 3D model of the vocal tract shown in figure $3 b$. 

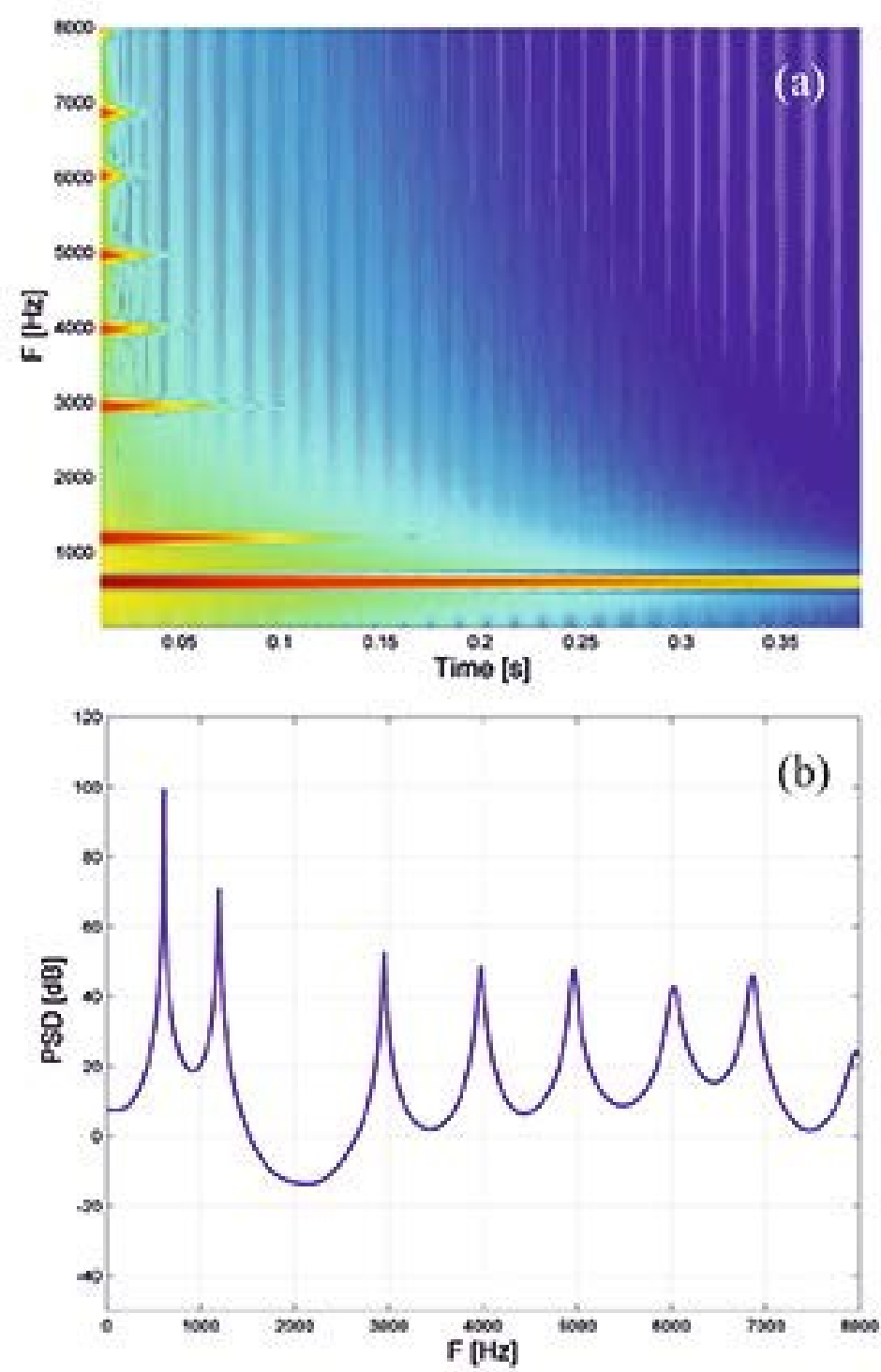

Figure 7. Numerically simulated spectrogram of the acoustic pressure computed at $10 \mathrm{~cm}$ in front of the mouth (upper panel) and the power spectral density of the response (lower panel) excited by a short airflow velocity pulse at the vocal folds using the simplified "1D" symmetric model of the vocal tract shown in figure 3c. 


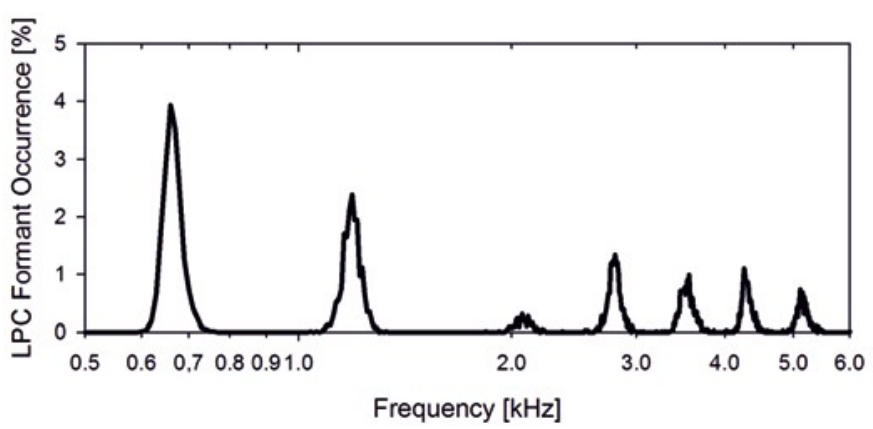

Figure 8. LPC formant histogram for the audio recording of the sustained [a:] vowel produced by the subject in the CT device. 

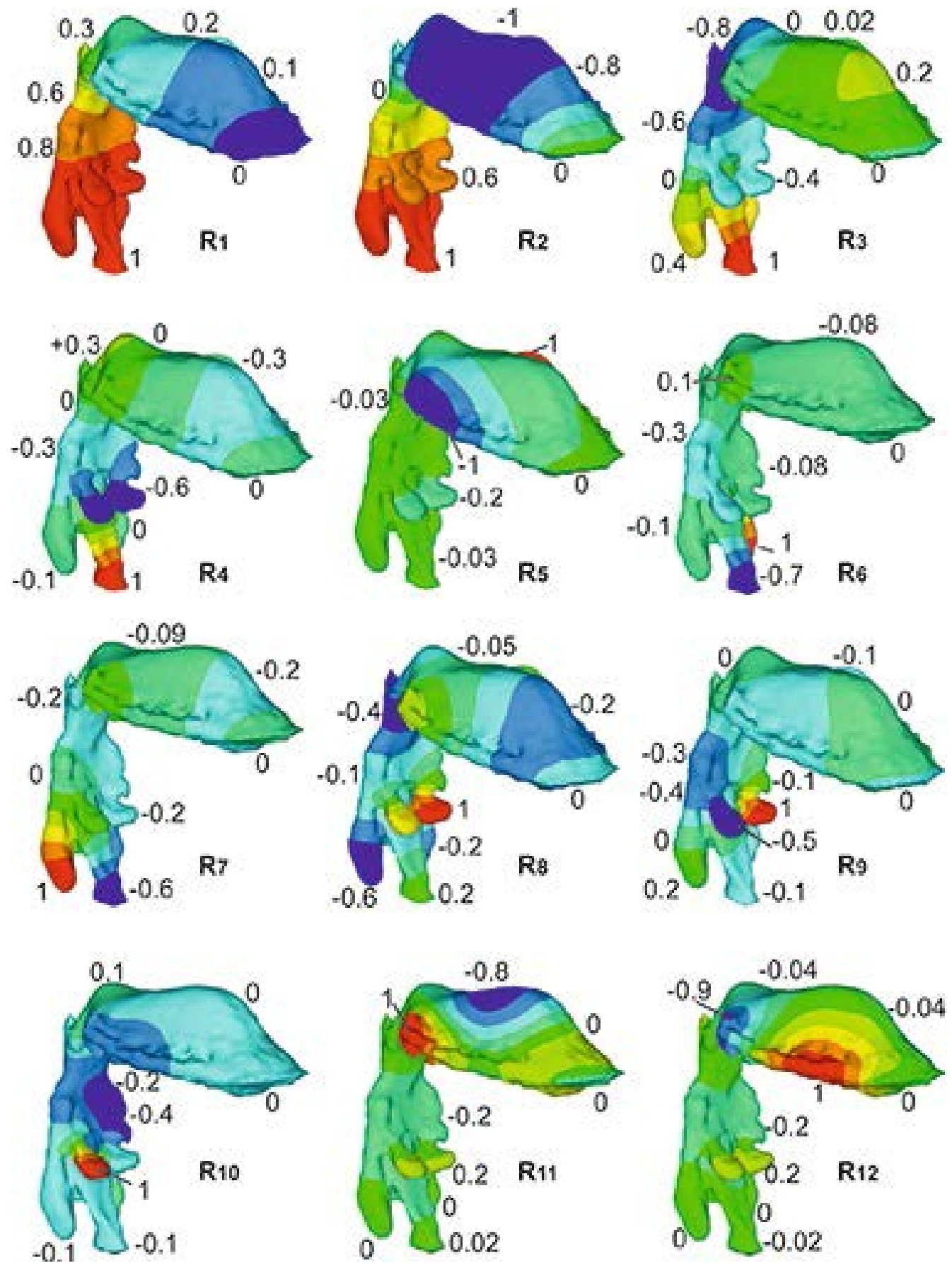

Figure 9. Computed acoustic mode shapes of vibrations for resonance frequencies $R_{1}-R_{12}$ of the vocal tract FE model for vowel [a:]. The pressure amplitudes are normalized for maximal values in each mode. 

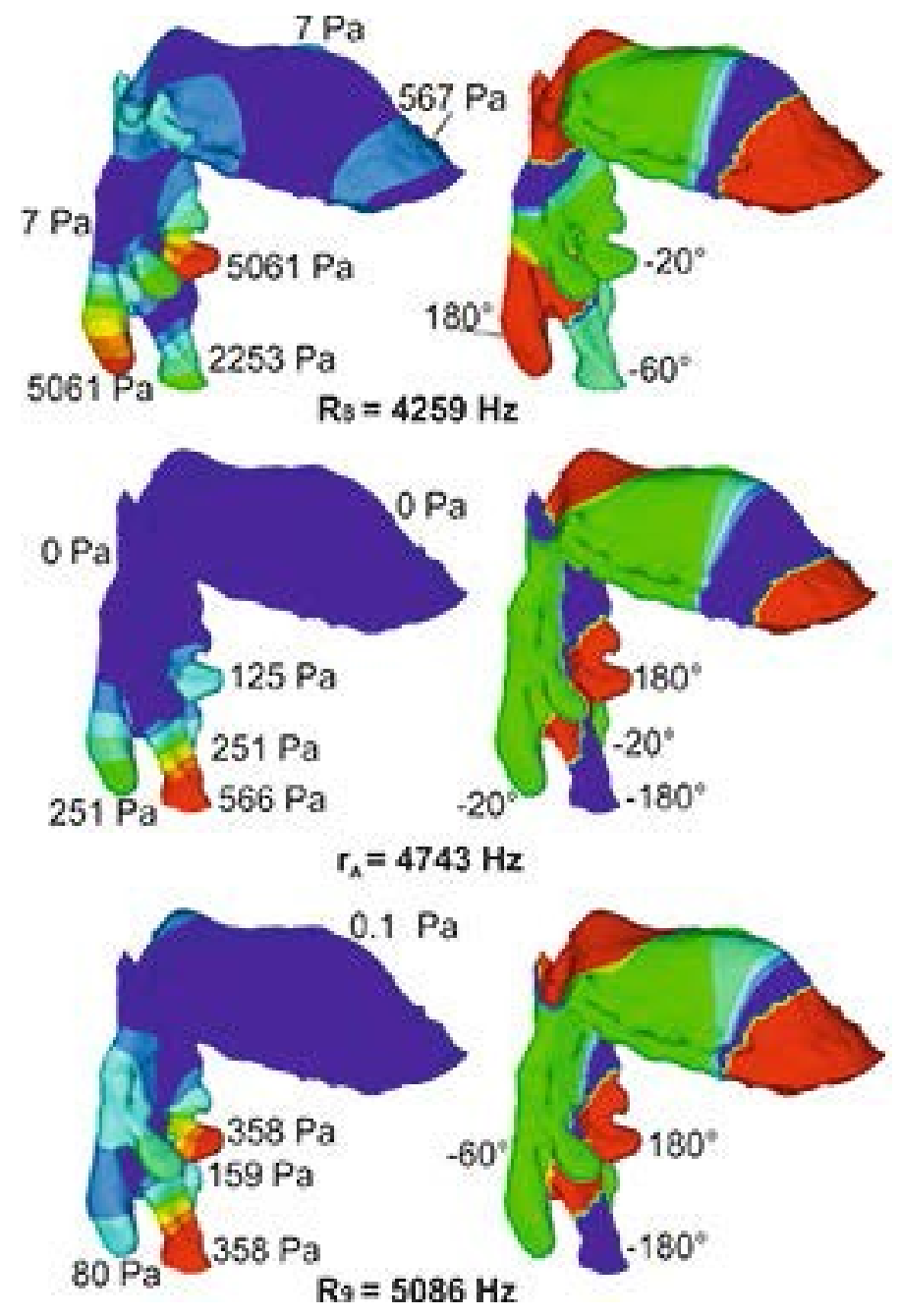

Figure 10. Absolute values of the acoustic pressure inside the vocal tract (left) and the corresponding phase shifts (right) between the excitation harmonic signal and the response for the resonance frequencies $R_{8}, R_{9}$ and at the main antiresonance $r_{A}=4743 \mathrm{~Hz}$ in between. 
Table I. Comparison of the formants obtained from an LPC analysis of the captured audio signals with the resonance frequencies (eigenfrequencies) computed by the modal analysis of the FE model.

\begin{tabular}{|l|c|l|l|l|l|}
\hline & $F_{0}(\mathrm{~Hz})$ & $F_{1}(\mathrm{~Hz})$ & $F_{2}(\mathrm{~Hz})$ & $F_{3}(\mathrm{~Hz})$ & $F_{4}(\mathrm{~Hz})$ \\
\hline LPC analysis & 156 & 660 & 1190 & 2800 & 3560 \\
\hline FEM & - & 649 & 1294 & 2785 & 3489 \\
\hline $\begin{array}{l}\text { Difference } \\
(\%)\end{array}$ & - & $-1,7$ & 8,0 & $-0,5$ & $-2,0$ \\
\hline
\end{tabular}

